\title{
Clofazimine is a broad-spectrum coronavirus inhibitor that antagonizes SARS-CoV-2 replication in primary human cell culture and hamsters
}

\section{Shuofeng Yuan}

University of Hong Kong

Xin Yin

Sanford Burnham Prebys Medical Discovery Institute https://orcid.org/0000-0003-2357-6718

\section{XiangZhi Meng}

University of Hong Kong

Jasper Chan

The University of Hong Kong

\section{Zi-Wei Ye}

State Key Laboratory of Emerging Infectious Diseases, Carol Yu Centre for Infection, Department of Microbiology, Li Ka Shing Faculty of Medicine, The University of Hong Kong

\section{Laura Riva}

Sanford Burnham Prebys Medical Discovery Institute

\section{Lars Pache}

Sanford Burnham Prebys Medical Discovery Institute https://orcid.org/0000-0002-4193-1042

\section{Chris Chun-Yiu Chan}

University of Hong Kong

\section{Pok-Man Lai}

University of Hong Kong

\section{Chris Chan}

The University of Hong Kong

\section{Vincent Poon}

The University of Hong Kong

\section{Naoko Matsunaga}

Sanford Burnham Prebys Medical Discovery Institute

\section{Yuan $\mathrm{Pu}$}

Sanford Burnham Prebys Medical Discovery Institute

\section{Chun-Kit Yuen}

University of Hong Kong

Jianli Cao

The University of Hong Kong 


\section{Ronghui Liang}

University of Hong Kong

\section{Kaiming Tang}

University of Hong Kong

\section{Li Sheng}

UCLA

\section{Yushen Du}

Department of Molecular and Medical Pharmacology, David Geffen School of Medicine, University of

California at Los Angeles, Los Angeles, California 90095, USA. https://orcid.org/0000-0002-8015-4207

\section{Wan Xu}

University of Hong Kong

\section{Kong-Hung Sze}

University of Hong Kong

Jinxia Zhang

Department of microbiology

\section{Hin Chu}

University of Hong Kong

\section{Kin-Hang Kok}

The University of Hong Kong https://orcid.org/0000-0003-3426-332X

\section{Kelvin To}

The University of Hong Kong https://orcid.org/0000-0002-1921-5824

\section{Dong-Yan Jin}

University of Hong Kong https://orcid.org/0000-0002-2778-3530

\section{Ren Sun}

The University of Hong Kong

\section{Sumit Chanda ( $\nabla$ schanda@sbpdiscovery.org)}

Sanford Burnham Prebys Medical Discovery Institute https://orcid.org/0000-0001-9399-7927

\section{Kwok-Yung Yuen}

University of Hong Kong https://orcid.org/0000-0002-2083-1552

\section{Biological Sciences - Article}

Keywords: Clofazimine, coronavirus inhibitor, pan-coronaviral inhibitory activity, COVID-19, coronavirus (CoV) outbreak

Posted Date: October 7th, 2020

DOI: https://doi.org/10.21203/rs.3.rs-86169/v1 
License: (c) (i) This work is licensed under a Creative Commons Attribution 4.0 International License. Read Full License

Version of Record: A version of this preprint was published at Nature on March 16th, 2021. See the published version at https://doi.org/10.1038/s41586-021-03431-4. 


\section{Abstract}

COVID-19 pandemic is the third zoonotic coronavirus (CoV) outbreak of the century after severe acute respiratory syndrome (SARS) in 2003 and Middle East respiratory syndrome (MERS) since 2012. Treatment options for CoVs are largely lacking. Here, we show that clofazimine, an anti-leprosy drug with a favorable safety and pharmacokinetics profile, possesses pan-coronaviral inhibitory activity, and can antagonize SARS-CoV-2 replication in multiple in vitro systems, including the human embryonic stem cellderived cardiomyocytes and ex vivo lung cultures. The FDA-approved molecule was found to inhibit multiple steps of viral replication, suggesting multiple underlying antiviral mechanisms. In a hamster model of SARS-CoV-2 pathogenesis, prophylactic or therapeutic administration of clofazimine significantly reduced viral load in the lung and fecal viral shedding, and also prevented cytokine storm associated with viral infection. Additionally, clofazimine exhibited synergy when administered with remdesivir. Since clofazimine is orally bioavailable and has a comparatively low manufacturing cost, it is an attractive clinical candidate for outpatient treatment and remdesivir-based combinatorial therapy for hospitalized COVID-19 patients, particularly in developing countries. Taken together, our data provide evidence that clofazimine may have a role in the control of the current pandemic SARS-CoV-2, endemic MERS-CoV in the Middle East, and, possibly most importantly, emerging CoVs of the future.

\section{Introduction}

The current pandemic of novel Coronavirus Disease 2019 (COVID-19) caused by severe acute respiratory syndrome coronavirus 2 (SARS-CoV-2) represents a global public health crisis. SARS-CoV-2 infection in human has a broad clinical spectrum ranging from mild to severe cases, with a mortality rate of $\sim 6.4 \%$ worldwide ${ }^{1}$. As of September 29, 2020, over 33 million cases had been reported in 235 countries, areas or territories with more than 1 million deaths, whereas a sizable portion of infected but non-symptomatic people with potential of transmissibility was also reported ${ }^{2}$.

The genetically diverse coronavirus (CoV) family, currently composed of four genera ( $a, \beta, \gamma$, and $\delta)$, infects birds, bats and a variety of mammals ${ }^{3}$. Within a decade, the world's human population has undergone three major CoV outbreaks. SARS-CoV-1 emerged in Guangdong, China in 2002 and, with the aid of commercial air travel, spread rapidly and globally, causing more than 8,000 cases with $10 \%$ mortality ${ }^{4}$. In 2012, MERS-CoV may have evolved to infect humans through bats by way of an intermediate camel host, causing over 1,700 cases with almost $40 \%$ mortality, and, like SARS-CoV-1, air travel has fueled global spread to 27 countries ${ }^{5}$.

Currently, there are no widely available specific antiviral therapies for CoV in humans. Remdesivir exhibited pan-coronavirus inhibitory potential ${ }^{6}$, and was recently granted emergency use authorization by the FDA for the treatment of COVID-19 based on the significant reduced time to recovery ${ }^{7}$. However, the therapy is far from optimal, particularly for severe COVID-19 patients, and can only be administered intravenously to hospitalized patients ${ }^{8,9}$. Thus development of additional therapeutic options is urgent, 
as well as the establishment of combinatorial regimens, such as the triple antiviral combination of interferon beta-1b, lopinavir-ritonavir, and ribavirin, which has been shown to be beneficial in a clinical trial ${ }^{10}$.

In efforts to accelerate the development of novel therapies for COVID-19, we previously profiled a library of known drugs encompassing approximately 12,000 clinical-stage or FDA-approved small molecules ${ }^{11}$. In this study, we focused on the antiviral mechanisms of action and in vivo efficacy of clofazimine, an FDA-approved molecule discovered as an anti-tuberculosis drug in 1957 and later used for treatment of leprosy ${ }^{12}$. Treating tuberculosis, clofazimine exhibits a minimum inhibitory concentration of $0.016 \mu \mathrm{g} / \mathrm{ml}$ (equivalent to $33.80 \mathrm{nM}$ ). The effective concentration of clofazimine against SARS-CoV-2 (half maximal effective concentration $310 \mathrm{nM}$ ) is clinically achievable with standard dosage in patients (peak serum concentration $861 \mathrm{nM})^{13}$. Here, we report the capability of clofazimine to confer protection against SARS-CoV-2 infection in primary human cell and animal models. Most importantly, clofazimine is affordable by COVID-19 patients in developing countries which may substantially relieve the critical care pressure caused by continuing pandemic ${ }^{14}$.

\section{Results}

\section{Clofazimine Inhibits SARS-CoV-2 and MERS-CoV Replication in Human Cellular Models}

Clofazimine has been found to be well tolerated in humans, showing a desirable safety profile at doses of $200 \mathrm{mg} /$ day in human ${ }^{13}$, a $\mathrm{C}_{\max }$ of $>861 \mathrm{nM}$, and a selectivity index $\left(\mathrm{CC}_{50} / \mathrm{EC}_{50}\right)$ around $30 \sim 50$ against SARS-CoV-2 infection ${ }^{15}$ (Figure 1a). These data suggest that therapeutic dosing of clofazimine may be achievable in patients at concentrations likely to have in vivo antiviral activity. Using SARS-CoV-2 infection as a model, we further characterized the antiviral activity of clofazimine in human embryonic stem cell-derived cardiomyocytes that robustly support SARS-CoV-2 replication ${ }^{16}$. Strikingly, and in a dose-dependent manner, clofazimine treatment reduced viral titers in the cell lysate by $>3-\log 10$ at a

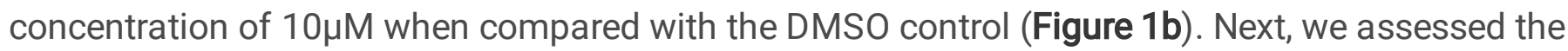
antiviral activity of clofazimine in an ex vivo lung culture system. Donor lung tissue was infected with SARS-CoV-2 for $24 \mathrm{~h}$ with drug treatment starting at 2 hours post-inoculation (hpi). Our results revealed that clofazimine potently antagonized viral replication in tissues that reflect the primary site of SARSCoV-2 replication (Figure 1c). To explore whether clofazimine confers protection against another epidemic CoV, we performed a plaque reduction assay for MERS-CoV. Clofazimine reduced MERS-CoV replication in VeroE6 cells with an $\mathrm{EC}_{50}$ of $1.48 \pm 0.17 \mu \mathrm{M}$ (Figure 1d). Immunofluorescence staining for MERS-CoV NP illustrated dramatic suppression of virus infection upon clofazimine treatment (upper panel, Figure 1e), which is supported by the flow cytometry analysis that the percentage of MERS-CoVinfected cells after clofazimine treatment decreased from $44.6 \%$ (DMSO) to $23.0 \%$ (clofazimine) at $24 \mathrm{hpi}$ in Huh7 cells (lower panel, Figure 1e). Overall, clofazimine exhibited potent broad spectrum anti-CoV, and antagonized SARS-CoV-2 replication in human primary cell and ex vivo lung models. 


\section{Clofazimine Interferes with Multiple Steps of Virus Life Cycle}

To understand the impact of clofazimine on the virus life cycle, antiviral activity was first evaluated by a time-of-drug addition assay in a single infectious cycle. Treatment with clofazimine during inoculation strongly inhibited SARS-CoV-2 infection, indicating that clofazimine exerts inhibitory effect on viral entry. Intriguingly, clofazimine also blocked SARS-CoV-2 infection at a post-entry step as evidenced by the observed reduction of viral infection when clofazimine was added at 5 hpi (Figure 2a). To further evaluate the impact of clofazimine on viral entry, we employed vesicular stomatitis virus (VSV)-based SARS-CoV-2 Spike (S) pseudotyped virions. Clofazimine treatment dramatically reduced the infectivity of both SARSCoV-1 S and SARS-CoV-2 S pseudotyped virions in VeroE6 cells. Interestingly, clofazimine did not impact MERS-CoV S pseudotyped virus particles (Figure $2 \mathbf{b}$ ), and this lack of entry inhibition may contribute to a lower potency observed for MERS-CoV. To confirm whether clofazimine also inhibits post-entry steps of viral replication, we evaluated the impact of clofazimine on viral RNA production by electroporating in vitro transcribed viral RNA into VeroE6 cells, which bypasses clofazimine-mediated inhibition on the entry process, and directly measures RNA synthesis (Figure 2c). As expected, remdesivir could effectively reduce the synthesis of negative-stranded RNA in a dose-dependent manner (Figure 2d). Intriguingly, viral RNA levels were also reduced by $1 \sim 1.5$ logs in the cells treated with clofazimine at concentrations above $5 \mu \mathrm{M}$ (Figure 2e). However, no significant effect was observed on electroporated GFP mRNA translation (Figure 2f). Collectively, these results demonstrated that clofazimine inhibit multiple steps in SARS-CoV-2 replication by interfering with spike-mediated entry as well as viral RNA replication.

\section{Transcriptional Analysis of Clofazimine Treatment}

To explore what is the impact of clofazimine on the transcriptional response of host cells, we employed RNA-Seq to profile the transcriptomic-wide changes during clofazimine treatment. We found that in human colorectal Caco-2 cells, clofazimine exhibited comparable anti-SARS-CoV-2 potency as that of remdesivir (Figure 3a), which was chosen for the downstream analysis. Transcriptional analysis was performed in Caco-2 cells which were either infected with SARS-CoV-2, treated with clofazimine $(10 \mu \mathrm{M})$ or both. Principal Component Analysis (PCA) on RNA-Seq results suggested that at $3 \mathrm{hpi}$, clofazimine treatment (3hpi. CFZ) caused overall transcriptome shift towards mock-infection group when compared with the vehicle control group (3 hpi) (Figure $3 b$ ), which is consistent with our data indicating that the drug inhibits viral infection at early time point post infection (Figure 2). At 6 hpi, there were 607 and 448 genes up- and down-regulated by SARS-CoV-2 infection, respectively (FDR $<0.05$, fold change $>2$ or $<0.5$ compared with mock). The RNA level of more than $90 \%$ of these genes was reverted by clofazimine treatment, indicating that clofazimine treatment abrogated transcriptomic changes caused by SARS-CoV2 infection. This is consistent with the PCA plot that treatment with clofazimine at 6 hpi (6 hpi. CFZ) caused a dramatic shift towards mock (Figure 3b, Extended Data Figure 1a). Interestingly, clofazimine treatment in the absence of infection (6h. CFZ) up-regulated genes that were enriched into innate immunity-related pathways, including MAPK, interleukin and TNF responses (Figure $3 c$ and $3 d$, Extended

Data Figure 1b). Particularly, transcription factors critical for immediate-early cellular response, including AP-1, SMAD, MAFF families, were upregulated by clofazimine (Figure 3c). When clofazimine was applied 
onto infected cells, most of these innate immune pathways were further enriched in upregulated genes (6hpi. CFZ, Figure 3d, Extended Data Figure 1b and 1c). These results suggest that clofazimine rewires the transcriptional landscape to prime the innate immunity-related pathways. While deficient early stage innate immune responses have been attributed to poor disease outcome, additional studies are required to determine if this enhanced antiviral response contributes to the in vitro and in vivo efficacy of the drug 17-19.

\section{Prophylactic and Therapeutic Treatment with Clofazimine Reduces SARS-CoV-2 Disease}

Clofazimine is useful for the treatment of disease due to multidrug resistant Mycobacterium tuberculosis, as well as leprosy and certain chronic skin diseases ${ }^{13}$.Previous pharmacokinetics studies revealed that clofazimine absorption varies from 45 to $62 \%$ following oral administration in leprosy patients. Coadministration of a $200 \mathrm{mg}$ dose of clofazimine with food resulted in a $\mathrm{C}_{\max }$ of $0.41 \mathrm{mg} / \mathrm{L}$ (equivalent to $861 \mathrm{nM}$ ) with a $T_{\max }$ of $8 \mathrm{~h}$. Administered in a fasting state, however, the corresponding $C_{\max }$ of clofazimine was $30 \%$ lower while the time to $C_{\max }$ was $12 \mathrm{~h}^{20}$. Intriguingly, clofazimine exerts antiinflammatory properties due to the suppression of macrophage activity, which may further mitigate the cytokine storm of SARS-CoV-2 infection in addition to its direct antiviral effects ${ }^{21}$. To determine the in vivo antiviral efficacy of clofazimine, we employed a golden Syrian hamster model that serves as a suitable tool to study antiviral effects and disease pathogenesis ${ }^{22}$. Since administration of clofazimine with a high fat meal provides better bioavailability ${ }^{13}$, we delivered the drug through oral route utilizing corn oil as vehicle. $25 \mathrm{mg} / \mathrm{kg} /$ day of clofazimine given on 3 consecutive days exhibited no significant observable toxicity to the animals. Remdesivir was included as a positive control drug and dosed at 15 $\mathrm{mg} / \mathrm{kg} /$ day based on its effective dosage in SARS-CoV-infected mice ${ }^{6}$.

Clofazimine has a relatively long duration of action with the mean elimination half-life approximately 25 days, thus we performed prophylactic treatment of hamsters with clofazimine before intranasally challenged with $10^{5} \mathrm{PFU}$ of SARS-CoV-2 (Figure 4a). Expectedly, the DMSO-treated control hamsters developed the clinical signs of lethargy, hunched back posture, and rapid breathing starting from $2 \mathrm{dpi}$, whereas the hamsters treated with clofazimine did not develop any clinical signs. At $2 \mathrm{dpi}$ when the viral loads and histopathological changes were expected to be worse, clofazimine decreased virus plaque forming units in lung tissues by $\sim 1$ to 1.5 logs (Figure $4 b$ ). Consistently, suppression of SARS-CoV-2 viral load in hamster lungs was confirmed in the clofazimine-treated hamsters (Figure 4c). To explore if the presence of clofazimine in the gastrointestinal tract, after intragastric administration, would prevent SARS-CoV-2 shedding, animal feces were collected at 2 dpi for viral RNA detection. Significantly less $(p=0.0353)$ viral copies were detectable in clofazimine-treated group when compared with the DMSO group, indicating its potential to diminish fecal shedding of SARS-CoV-2 (Figure 4d). Increased proinflammatory cytokines and chemokines is associated with disease severity of COVID-19 patients. To ascertain if the therapeutic effect of clofazimine alleviates virus-induced cytokine dysregulation, we determined the expression levels of interleukin 6 (IL-6), tumor necrosis factor alpha (TNF-a), and C-C chemokine receptor type 4 (CCR4), which are prognostic markers for severe COVID-19 ${ }^{23}$. As shown in 
Figure 4e, mRNA expression of IL-6 ( $p=0.0001)$, TNF- $a(p=0.0006)$, and CCR4 $(p=0.0029)$ were remarkably reduced in the hamsters treated with clofazimine. Previous reports have shown that clofazimine can inhibit lymphocyte function ${ }^{24}$. To explore if this is the case in our animal model, hamster sera were collected at 14dpi for measurement of anti-NP antibody using an ELISA-based enzyme immunoassay. Apparently, similarly high levels of antibody responses were triggered in DMSO and clofazimine groups, indicating insignificant suppression of humoral immune response of $\mathrm{B}$ lymphocyte by clofazimine (Figure 4i). Taken together, prophylactic administration of clofazimine conferred protection against SARSCoV-2 challenge by reducing the virus replication and the associated inflammatory dysregulation.

To recapitulate the scenario that most COVID-19 patients will receive treatment after diagnosis or disease onset, it was of interest to determine whether therapeutic treatment of clofazimine, with the first dosing given $24 \mathrm{~h}$ after virus exposure, would also ameliorate SARS-CoV-2 disease. SARS-CoV-2 infected hamsters were given 3 doses in total before being scarified at $4 \mathrm{dpi}$ for lung viral yield detection. Generally, both therapeutic clofazimine and remdesivir suppressed virus lung titers when compared with the DMSO control (Figure $\mathbf{4 f}$ and $\mathbf{4 g}$ ). The diminished clinical signs were also associated with substantially decreased IL-6 protein amount in the clofazimine $(p=0.0119)$ and remdesivir-treated $(p=0.0074)$ hamster sera (Figure $4 h$ ), as increased serum IL-6 level has been correlated with respiratory failure and adverse clinical outcome ${ }^{25}$.

As for the severity of lung damage, histological examination of hematoxylin and eosin (H\&E) stained lung tissues was performed. Significant amelioration of lung damage was observed after clofazimine treatment (Figure 4j). For prophylactic administration, lung tissues from the DMSO group showed severe bronchiolar cell death with massive cell debris filling the lumen, alveolar wall thickened with alveolar exudation; whereas prophylaxis with clofazimine showed no apparent pathological changes. With therapeutic administration, DMSO-treated lung sections showed large areas of lung consolidation with alveolar infiltration and exudation, while clofazimine treated lungs exhibited a mild degree of alveolar wall thickening and capillary congestion. Generally, prophylactic administration conferred more dramatic improvements of lung pathology when compared with therapeutic administration, which might be attributed to the relatively long $T_{\max }$ of clofazimine. Nevertheless, both prophylactic and therapeutic treatment with clofazimine reduced SARS-CoV-2 disease in vivo.

\section{Clofazimine Exhibits Antiviral Synergy with Remdesivir}

Since the emergency use authorization by the US FDA, remdesivir is considered the standard of care for the treatment of COVID-19. To understand the impact of combinatorial treatments of remdesivir and clofazimine on SARS-CoV-2 replication, we conducted a matrixed dose response analysis. We found that co-application of clofazimine and remdesivir impacts SARS-CoV-2 replication in a manner that extends beyond the additive combinatorial activity predicted by the Bliss independence model (maximal Bliss Synergy Score of 44.28; Figure 5a, Extended Data Figure 2), and indicates these two drugs harbor a synergistic antiviral relationship. Clofazimine can be safely dosed at $200 \mathrm{mg} /$ day for the treatment of leprosy, which results in average serum concentrations of $1.79 \mu \mathrm{M}$, although the bioavailable fraction of 
the molecule will be a function of plasma protein binding. The addition of $1.25 \mu \mathrm{M}$ clofazimine in an in vitro cellular assay with a $10 \%$ concentration of FBS resulted in a nearly 20 -fold decrease in concentrations of remdesivir required to inhibit viral replication by $90 \%$ (Figure 5 b). Importantly, the combination of drugs did not elicit additional cellular cytotoxicity (Figure 5c).

\section{Discussion}

Clofazimine was first used to treat leprosy in 1969 , and gained FDA approval in $1996^{26}$. It is an orally bioavailable drug that is included in the WHO Model List of Essential Medicines. It is generally welltolerated, with adverse events that include skin discoloration, ichthyosis, and gastrointestinal intolerance 27. Besides treating leprosy, clofazimine is an intriguing medication that has implications for multi-drugresistant tuberculosis (MDR-TB) and extensively drug-resistant tuberculosis (XDR-TB). Showing good safety evidence, clofazimine is a part of WHO group $\mathrm{C}$ in terms of the treatment guidelines for MDR-TB ${ }^{28}$. This is supported by clinical trials in China, Bangladesh, and Brazil where patients were receiving clofazimine for 18 21 months at a dose of $100 \mathrm{mg} /$ day 29,30 .

We observed that clofazimine shows pleotropic antiviral activities against SARS-CoV-2, including inhibition of spike-dependent entry. While it has been reported that clofazimine is internalized through endocytosis, further investigation is required to elucidate if the drug directly impinges on endosomal function to inhibit viral entry ${ }^{31}$, and why the leprosy drug selectively blocks SARS-CoV, but not MERS-CoV, entry into cells. Importantly, this drug is a lipophilic rhimophenazine dye which inhibits mycobacteria through intercalation into bacterial DNA, likely inhibiting DNA replication and proliferation ${ }^{32}$. While we observe that clofazimine inhibits the RNA replication of SARS-CoV-2, additional studies are also required to determine if the drug similarly inhibits CoV RNA unwinding or template function.

In SARS-CoV-2 infection, a delayed innate immune response may result in uncontrollable cytokine storm 19,33. Clofazimine's effect on rewiring the transcriptional landscape of the cell towards an antiviral status may be important in a disease setting, and understanding the contribution of this activity toward in vivo disease amelioration can provide insight towards its potential to improve viral control through enhancement of innate immune activities. Paradoxically, clofazimine has been reported to possess antiinflammatory activity through the inhibition of macrophage function and $\mathrm{T}$ lymphocyte activation and proliferation ${ }^{34}$. Further elucidation of how clofazimine treatment may balance regulation of innate and adaptive immune responses to improve disease outcome will be important to understand its potential clinical efficacy.

In this study, a prophylaxis regimen with three daily doses substantially protected animal from SARS-CoV2 infection (Figure 4). In contrast to orally bioavailable clofazimine, remdesivir is currently given through intravenous administration, which makes it difficult to provide on an outpatient or prophylactic basis. Moreover, remdesivir requires a complex synthesis process to manufacture, resulting in a high treatment cost (US\$520 per vial, or US\$3,120 per treatment course) and availability for only several million patients 
over the next two years ${ }^{35}$. In view of the potentially-long epidemic dynamics and pressures on critical care capacity over the next 5 years, as well as the potential resurgence of SARS-CoV- 2 in the future, clofazimine, which only costs US\$1.43/100mg tablets, can be considered as one of the potential countermeasures for global control of the COVID-19 pandemic ${ }^{14}$, especially in developing countries. Additionally, co-administration with clofazimine could significantly reduce costs for remdesivir-based treatment of COVID-19, and extend worldwide supplies of remdesivir, and a combinatorial approach can also help mitigate the emergence of drug-resistant viral strains.

Clofazimine, in conjunction with interferon, is currently being evaluated in clinical trials for the treatment of COVID-19 (Trial Number: NCT04465695). The ex vivo and in vivo efficacy of clofazimine suggests that clinical evaluation of the drug as monotherapy in outpatient setting for treatment of early stage disease, or in combination with remdesivir in hospitalized patients, is critical for establishing its potential as a rapidly scalable treatment option for COVID-19.

\section{Materials And Methods}

\section{Cells and viruses}

Human hepatoma Huh7 (JCRB, 0403) cells and monkey VeroE6 cells (ATCC, CRL-592 1586) were maintained in DMEM culture medium supplemented with $10 \%$ heat-inactivated $\mathrm{FBS}, 50 \mathrm{U} / \mathrm{ml}$ penicillin and $50 \mu \mathrm{g} / \mathrm{ml}$ streptomycin. Ventricular cardiomyocyte were differentiated from the human embryonic stem cell HES2 (ESI) maintained in mTeSR1 medium (STEMCELL Technologies) ${ }^{36}$. Briefly, HES2 cells were dissociated with Accutase (Invitrogen) into single cells suspensions on day 0. Cells were seeded on low-attachment culture vessels (Corning) and cultured in mTeSR 1 medium supplemented with $40 \mu \mathrm{g} / \mathrm{ml}$

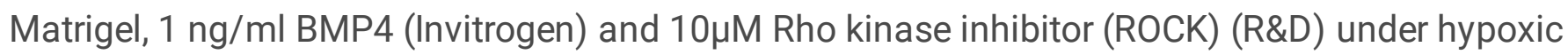
environment with $5 \% \mathrm{O}_{2}$. From day 1 to 3 , cells were cultured in StemPro34 SFM (Invitrogen) with 50 $\mu \mathrm{g} / \mathrm{ml}$ ascorbic acid (AA) (Sigma), $2 \mathrm{mM}$ Gluta-MAX (Invitrogen), $10 \mathrm{ng} / \mathrm{ml} \mathrm{BMP4}$, and $10 \mathrm{ng} / \mathrm{ml}$ human recombinant activin-A (Invitrogen). From day 4 to day 7, $5 \mu \mathrm{M}$ Wnt inhibitor IWR-1(Tocris) was added. From day 8 to day 14, cells were cultured under normoxia in RPMI 1640 medium (Invitrogen) supplemented with $2 \mathrm{mM}$ Gluta-MAX, 1×B-27 supplement (Invitrogen) and $50 \mu \mathrm{g} / \mathrm{mL}$ AA. The cells were then dissociated with Accutase and seeded as monolayer in desired culture vessels for 3 days before infections. The SARS-CoV-2 HKU-001a strain (GenBank accession number: MT230904) was isolated from the nasopharyngeal aspirate specimen of a laboratory-confirmed COVID-19 patient in Hong Kong 22. The SARS-CoV-2, Isolate USA-WA1/2020 was deposited by the Centers for Disease Control and Prevention and obtained through BEI Resources. The MERS-CoV (HCoV-EMC/2012) was a gift from Dr. Ron Fouchier. All experiments involving live SARS-CoV-2 and MERS-CoV followed the approved standard operating procedures of the Biosafety Level 3 facility at the University of Hong Kong as we previously described ${ }^{37}$.

\section{Antiviral evaluation in human ex vivo lung tissues}


Human lung tissues for ex vivo studies were obtained from patients undergoing surgical operations at Queen Mary Hospital, Hong Kong as previously described ${ }^{38}$. The donors gave written consent as approved by the Institutional Review Board of the University of Hong Kong/Hospital Authority Hong Kong West Cluster (UW13-364). The freshly obtained lung tissues were processed into small rectangular pieces and were rinsed with advanced DMEM/F12 medium (Gibco) supplemented with $2 \mathrm{mM}$ of HEPES (Gibco), $1 \times$ GlutaMAX (Gibco), $100 \mathrm{U} / \mathrm{ml}$ penicillin, and $100 \mu \mathrm{g} / \mathrm{mL}$ streptomycin. The specimens were infected with SARS-CoV-2 HKU-001a with an inoculum of $1 \times 10^{6} \mathrm{PFU} / \mathrm{ml}$ at $500 \mu \mathrm{L}$ per well. After two hours, the inoculum was removed, and the specimens were washed 3 times with PBS. The infected human lung tissues were then cultured in $1 \mathrm{ml}$ of advanced DMEM/F12 medium with $2 \mathrm{mM}$ HEPES (Gibco), 1×GlutaMAX (Gibco), $100 \mathrm{U} / \mathrm{mL}$ penicillin, $100 \mu \mathrm{g} / \mathrm{mL}$ streptomycin, $20 \mu \mathrm{g} / \mathrm{mL}$ vancomycin, $20 \mu \mathrm{g} / \mathrm{mL}$ ciprofloxacin, $50 \mu \mathrm{g} / \mathrm{mL}$ amikacin, and $50 \mu \mathrm{g} / \mathrm{mL}$ nystatin. Supernatants were collected at 24 hours post inoculation (hpi) for plaque assays.

\section{Antiviral assessment in a SARS-CoV-2 infected hamster model}

Male and female Syrian hamster, aged 6-10 weeks old, were kept in biosafety level 2 housing and given access to standard pellet feed and water ad libitum as we previously described ${ }^{22}$. All experimental protocols were approved by the Animal Ethics Committee in the University of Hong Kong (CULATR) and were performed according to the standard operating procedures of the biosafety level 3 animal facilities (Reference code: CULATR 5370-20). Experimentally, each hamster was intranasally inoculated with $10^{5}$ PFU of SARS-CoV-2 in $100 \mu \mathrm{L}$ PBS under intraperitoneal ketamine $(200 \mathrm{mg} / \mathrm{kg})$ and xylazine $(10 \mathrm{mg} / \mathrm{kg})$ anesthesia. Prophylactic treatment used oral administration of clofazimine given on $-3,-2$ and $-1 \mathrm{dpi}$ (25 $\mathrm{mg} / \mathrm{kg} /$ day), followed by virus challenge at $0 \mathrm{dpi}$, while therapeutic post-exposure and oral administration of clofazimine were performed on 1, 2, and $3 \mathrm{dpi}(25 \mathrm{mg} / \mathrm{kg} /$ day) with the first dosage given at $24 \mathrm{hpi}$. Clofazimine was delivered using corn oil (Sigma-Aldrich, C8267) as vehicle. Remdesivir (15 mg/kg/day, MedChemExpress) was used as a positive control through intraperitoneal injection. One percent DMSO in corn oil was used as a placebo control through oral route. Animals were sacrificed at $2 \mathrm{dpi}$ and $4 \mathrm{dpi}$ for virological and histopathological analyses. Viral yield in the lung tissue homogenates and/or feces were detected by plaque assay and/or qRT-PCR methods. Cytokine and chemokine profile of the hamster lungs were detected by $2^{-\triangle \Delta C T}$ method using probe-based one step qRT-PCR (Qiagen). ELISA kit was utilized to determine the Interleukin 6 (IL-6) amount in the hamster sera on 4 dpi according to the manufacture's recommendations (ELISAGenie, HMFI0001). Tissue pathology of infected animals was examined by H\&E staining in accordance to the established protocol ${ }^{39}$. On 14 dpi, enzyme immunoassay (EIA) was utilized to determine the antibody titer of hamster sera against SARS-CoV-2 NP antigen ${ }^{40}$. Briefly, 96-well immune-plates (Nunc) were coated with $100 \mu \mathrm{L} /$ well $\left(0.1 \mu \mathrm{g} /\right.$ well) of SARS-CoV-2 NP in $0.05 \mathrm{M} \mathrm{NaHCO}_{3}$ $\left(\mathrm{pH}\right.$ 9.6) overnight at $4^{\circ} \mathrm{C}$. After blocking, $100 \mu \mathrm{L}$ of heat-inactivated serum samples were serial-diluted before adding to the wells and incubated at $37^{\circ} \mathrm{C}$ for $1 \mathrm{~h}$. The attached antibodies were detected using horseradish-peroxidase-conjugated rabbit anti-hamster IgG antibody (Invitrogen, A18895). The reaction was developed by adding diluted 3,3',5,5'-tetramethylbenzidine single solution (Invitrogen) and stopped with $0.3 \mathrm{~N} \mathrm{H}_{2} \mathrm{SO}_{4}$. The optical density (OD) was read at 450/620 nm using a microplate reader. 


\section{RNA-Seq analysis}

Fastq files from RNA-seq were quality examined by FastQC (https://www.bioinformatics.babraham.ac.uk/projects/fastqc/). Reads were processed by cutadapt to remove reads with low quality and to trim adapters. Trimmed reads were aligned to hg38 reference genome using Tophat2 ${ }^{41}$. Reads assigned to each gene were counted by featureCounts ${ }^{42}$ with refseq gene sets as references. Genes without at least 1 read mapped on average in each sample were considered undetectable and were filtered out. Read counts were normalized by Trimmed Mean of Mvalues (TMM) method and differential expression was calculated using R package edgeR and Genewise Negative Binomial Generalized Linear Models with Quasi-likelihood Tests (glmQLFit) method was used for statistical tests. Cut-offs imposed for differential expression analysis was set as False Discovery Rate (FDR) of 0.05 and fold change $>2$ or $<0.5$. The pathway analysis was performed by R package clusterProfiler ${ }^{43}$. Heatmaps were plotted using R package pheatmap (Kolde, R. (2013). pheatmap: Pretty Heatmaps. R package version 0.7.7. http://CRAN.R-project.org/package=pheatmap.). Other plots were generated by R package ggplot2 (Wickham H (2016). ggplot2: Elegant Graphics for Data Analysis. Springer-Verlag New York. ISBN 978-3-319-24277-4, https://ggplot2.tidyverse.org). The plots were made using Cytoscape ${ }^{44}$. PCA analysis was performed by R package factoextra.

\section{Pseudotyping of VSV and Pseudotype-based inhibition assay}

Vesicular Stomatitis Virus (VSV) pseudotyped with spike proteins of MERS-CoV, SARS-CoV-1, and SARSCoV-2 were generated as previously reported with some modifications ${ }^{45}$. Briefly, BHK-21/WI-2 cells (Kerafast, MA) overexpressing the spike proteins were inoculated with VSV-G pseudotyped $\triangle$ G-luciferase VSV (Kerafast, MA). After $2 \mathrm{~h}$ inoculation at $37^{\circ} \mathrm{C}$, the inoculum was removed and cells were refed with DMEM supplemented with 5\% FBS and VSV-G antibody (11, mouse hybridoma supernatant from CRL2700; ATCC). Pseudotyped particles were collected at $24 \mathrm{~h}$ post-inoculation, then centrifuged at $1,320 \times \mathrm{g}$ to remove cell debris and stored at $-80^{\circ} \mathrm{C}$ until use.

To determine the effect of the compounds on viral entry, VeroE6 cells were treated with clofazimine at a concentration of $2.5 \mu \mathrm{M}$ for $1 \mathrm{~h}$ prior to inoculation with respective pseudotyped VSV. After $2 \mathrm{~h}$ inoculation in the presence of the compounds, the inoculum was removed and cells were refed with fresh medium for further culture. The activity of firefly luciferase was measured using bright-Glo ${ }^{\text {TM }}$ luciferase assay (Promega) for quantitative determination at $16 \mathrm{~h}$ post-transduction.

\section{The effect of clofazimine on SARS-CoV-2 viral replication}

The full-length SARS-CoV-2 viral RNA transcripts were in vitro synthesized from an infectious clone of SARS-CoV-2 (kindly provided by Pei-Yong Shi, UTMB ) according to a recently published protocol ${ }^{46} .10 \mu \mathrm{g}$ of total RNA transcripts and $5 \mu \mathrm{g}$ SARS-CoV-2 NP gene transcript were mixed with VeroE6 cells stably expressing SARS-CoV-2 NP protein and then added into a $0.2 \mathrm{~cm}$ cuvette for nucleofection with the 4DNucleofectorTM Core Unit (Lonza) using pulse code V-001. Immediately after electroporation, $1000 \mu \mathrm{L}$ of 
pre-warmed media was added to the cuvette and cells were subsequently aliquoted into 384-well plates. Two hours post-seeding, compounds at different concentrations were added into each well. At 12 hours post-electroporation, intracellular and viral RNA was purified from the treated cells with TurboCapture 384 mRNA Kit (Qiagen) in accordance with the manufacturer's instructions. The purified RNA was subjected to first-strand cDNA synthesis using the high-capacity cDNA reverse transcription kit (Applied Biosystems, Inc) with the following primer (TagRdRp-F: 5'- CGGTCATGGTGGCGAATAACCCTGTGGGTTTTACACTTAA3'). Real-time PCR analysis was performed using TaqPath 1-step RT-qPCR Master Mix (Applied Biosystems, Inc). The following primers and probe were used for negative-stranded RNA detection: Tag-F: 5'-CGGTCATGGTGGCGAATAACCCTGT-3', ORF1ab-R: 5'-ACGATTGTGC ATCAGCTGA-3', ORF1ab-P: 5'-6FAM-CCGTCTGCGGTATGTGGAAAGGTTATGG -BHQ1-3').

\section{Time-of-addition assay}

Time-of-drug-addition assay was performed to investigate which stage of SARS-CoV-2 life cycle clofazimine interfered with as previously described ${ }^{47}$. Briefly, VeroE6 cells were seeded in 96 -well plates ( $4 \times 10^{4}$ cells/well). The cells were infected by SARS-CoV-2 USA-WA1/2020 at an MOI of 1.5 and then incubated for additional $1 \mathrm{~h}$. The viral inoculum was then removed and the cells were washed twice with PBS. At 1 hpi (i.e., post entry), clofazimine at a concentration of $5 \mu \mathrm{M}$ was added to the infected cells at time-points indicated, followed by the incubation at $37^{\circ} \mathrm{C}$ in $5 \% \mathrm{CO} 2$ until $10 \mathrm{hpi}$ (i.e. one virus life cycle). Cells were fixed at $10 \mathrm{hpi}$ for quantification of the percentage of infected cells using an immunofluorescence assay targeting SARS-CoV-2 NP.

\section{Data availability}

Complete sequences of SARS-CoV-2 HKU-001a and SARS-CoV-2 USA-WA1/2020 are available through GenBank (accession numbers MT230904 (HKU-001a), MT246667 and MN908947 (USA-WA1/2020)). The raw RNA-Seq data reported in Figure 3 have been deposited in GEO. Other supporting raw data are available from the corresponding author upon reasonable request. Source data are provided with this paper.

\section{Declarations}

\section{Acknowledgment}

The authors acknowledge the assistance of the University of Hong Kong Li Ka Shing Faculty of Medicine Centre for PanorOmic Sciences. This study was partly supported by funding to University of Hong Kong: the National Key R\&D Programmes of China (grants 2020YFA0707500 and 2020YFA0707504) and donations from the Lo Ying Shek Chi Wai Foundation, Richard Yu and Carol Yu, the Shaw Foundation of Hong Kong, Michael Seak-Kan Tong, May Tam Mak Mei Yin, Hui Ming, Hui Hoy and Chow Sin Lan Charity Fund Limited, Chan Yin Chuen Memorial Charitable Foundation, Marina ManWai Lee, the Hong Kong Hainan Commercial Association South China Microbiology Research Fund, the Jessie \& George Ho Charitable Foundation, Perfect Shape Medical Limited, Kai Chong Tong, Foo Oi 
Foundation Limited, Tse Kam Ming Laurence, and Norman \& Cecilia Yip Foundation. This work was also supported by the grants to the Sanford Burnham Prebys Medical Discovery Institute: DoD: W81XWH-20-10270; DHIPC: U19 Al118610; Fluomics/NOSI: U19 Al135972, as well as generous philanthropic donations from Dinah Ruch and Susan \& James Blair. This research was also partly funded by CRIP (Center for Research for Influenza Pathogenesis), a NIAID supported Center of Excellence for Influenza Research and Surveillance (CEIRS, contract \# HHSN272201400008C), by DARPA grant HR0011-19-2-0020, by an administrative supplement to NIAID grant U19AI142733, and by the generous support of the JPB Foundation, the Open Philanthropy Project (research grant 2020-215611 (5384)). The funding sources had no role in the study design, data collection, analysis, interpretation, or writing of the report.

\section{Author contributions}

S.Y., X.Y., X.M., Z.W.Y., L.R., N.M., L.P., Y.P., R.L., C.C.Y.C., P.M.L., J.C., R.H.L., and W.X. designed and/or performed experiments. S.Y., X.Y., X.M., Z.W.Y., J.F.W.C., L.P., Y.D., K.H.S., and H.C. analyzed data. S.Y., X.Y., Z.W.Y., C.K.Y., K.H.K., K.Y.S, W.K. A and L.S. generated critical reagents. K.Y.Y., S.K.C., R.S., S.Y., X.Y., X.M., and L.P. wrote the manuscript. S.Y., J.F.W.C., C.C.S.C., V.K.M.P. and K.T. provided the animal data. A.J.Z. and J.F.W.C. performed histopathological evaluation. S.K.C., R.S. I.F.N.H., R.A.L. and D.Y.J. provided conceptual advice and troubleshooting. K.Y.Y., S.K.C. and R.S. oversaw the conception and supervised the study. K.Y.Y. and S.K.C. provided the grant support.

\section{Competing interests}

J.F.W.C. has received travel grants from Pfizer Corporation Hong Kong and Astellas Pharma Hong Kong Corporation Limited and was an invited speaker for Gilead Sciences Hong Kong Limited and Luminex Corporation. S.K.C. are inventors on a patent application on repurposed antiviral compounds for SARSCoV-2 owned by Scripps Research and Sanford Burnham Prebys. US Patent Application Serial No. 63/010630, entitled Methods and Compositions for Antiviral Treatment relates to aspects of this work and was filed on 15 April 2020. The corresponding authors had full access to all the data in the study and had final responsibility for the decision to submit for publication. The other authors declare no competing interests.

\section{References}

1 Yuen, K.-S., Ye, Z.-W., Fung, S.-Y., Chan, C.-P. \& Jin, D.-Y. SARS-CoV-2 and COVID-19: The most important research questions. Cell Biosci 10, 40-40, doi:10.1186/s13578-020-00404-4 (2020).

2 Lee, S. et al. Clinical Course and Molecular Viral Shedding Among Asymptomatic and Symptomatic Patients With SARS-CoV-2 Infection in a Community Treatment Center in the Republic of Korea. JAMA Internal Medicine, doi:10.1001/jamainternmed.2020.3862 (2020).

3 Chan, J. F. et al. Middle East respiratory syndrome coronavirus: another zoonotic betacoronavirus causing SARS-like disease. Clin Microbiol Rev 28, 465-522, doi:10.1128/CMR.00102-14 (2015). 
4 Cheng, V. C., Lau, S. K., Woo, P. C. \& Yuen, K. Y. Severe acute respiratory syndrome coronavirus as an agent of emerging and reemerging infection. Clin Microbiol Rev 20, 660-694, doi:10.1128/CMR.0002307 (2007).

5 de Wit, E., van Doremalen, N., Falzarano, D. \& Munster, V. J. SARS and MERS: recent insights into emerging coronaviruses. Nat Rev Microbiol 14, 523-534, doi:10.1038/nrmicro.2016.81 (2016).

6 Sheahan, T. P. et al. Broad-spectrum antiviral GS-5734 inhibits both epidemic and zoonotic coronaviruses. Sci Trans/ Med 9, doi:10.1126/scitransImed.aal3653 (2017).

7 Beigel, J. H. et al. Remdesivir for the Treatment of Covid-19 - Preliminary Report. N Engl J Med, doi:10.1056/NEJMoa2007764 (2020).

8 Wang, Y. et al. Remdesivir in adults with severe COVID-19: a randomised, double-blind, placebocontrolled, multicentre trial. Lancet 395, 1569-1578, doi:10.1016/S0140-6736(20)31022-9 (2020).

9 Goldman, J. D. et al. Remdesivir for 5 or 10 Days in Patients with Severe Covid-19. N Engl J Med, doi:10.1056/NEJMoa2015301 (2020).

10 Hung, I. F. et al. Triple combination of interferon beta-1b, lopinavir-ritonavir, and ribavirin in the treatment of patients admitted to hospital with COVID-19: an open-label, randomised, phase 2 trial. Lancet 395, 1695-1704, doi:10.1016/S0140-6736(20)31042-4 (2020).

11 Riva, L. et al. A Large-scale Drug Repositioning Survey for SARS-CoV-2 Antivirals. bioRxiv, doi:10.1101/2020.04.16.044016 (2020).

12 Gopal, M., Padayatchi, N., Metcalfe, J. Z. \& O'Donnell, M. R. Systematic review of clofazimine for the treatment of drug-resistant tuberculosis. Int J Tuberc Lung Dis 17, 1001-1007, doi:10.5588/ijtld.12.0144 (2013).

13 Nix, D. E. et al. Pharmacokinetics and relative bioavailability of clofazimine in relation to food, orange juice and antacid. Tuberculosis (Edinb) 84, 365-373, doi:10.1016/j.tube.2004.04.001 (2004).

14 Kissler, S. M., Tedijanto, C., Goldstein, E., Grad, Y. H. \& Lipsitch, M. Projecting the transmission dynamics of SARS-CoV-2 through the postpandemic period. Science $368,860-868$, doi:10.1126/science.abb5793 (2020).

15 Riva, L. et al. Discovery of SARS-CoV-2 antiviral drugs through large-scale compound repurposing. Nature, doi:10.1038/s41586-020-2577-1 (2020).

16 Sharma, A. et al. Human iPSC-Derived Cardiomyocytes Are Susceptible to SARS-CoV-2 Infection. Cell Rep Med 1, 100052-100052, doi:10.1016/j.xcrm.2020.100052 (2020). 
17 Bastard, P. et al. Auto-antibodies against type I IFNs in patients with life-threatening COVID-19. Science, eabd4585, doi:10.1126/science.abd4585 (2020).

18 Zhang, Q. et al. Inborn errors of type I IFN immunity in patients with life-threatening COVID-19. Science, eabd4570, doi:10.1126/science.abd4570 (2020).

19 Hadjadj, J. et al. Impaired type I interferon activity and inflammatory responses in severe COVID-19 patients. Science 369, 718-724, doi:10.1126/science.abc6027 (2020).

20 Feng, P. C., Fenselau, C. C. \& Jacobson, R. R. Metabolism of clofazimine in leprosy patients. Drug Metab Dispos 9, 521-524 (1981).

21 Yoon, G. S. et al. Clofazimine Biocrystal Accumulation in Macrophages Upregulates Interleukin 1 Receptor Antagonist Production To Induce a Systemic Anti-Inflammatory State. Antimicrob Agents Chemother 60, 3470-3479, doi:10.1128/AAC.00265-16 (2016).

22 Chan, J. F. et al. Simulation of the clinical and pathological manifestations of Coronavirus Disease 2019 (COVID-19) in golden Syrian hamster model: implications for disease pathogenesis and transmissibility. Clin Infect Dis, doi:10.1093/cid/ciaa325 (2020).

23 Ulhaq, Z. S. \& Soraya, G. V. Interleukin-6 as a potential biomarker of COVID-19 progression. Med Mal Infect 50, 382-383, doi:10.1016/j.medmal.2020.04.002 (2020).

24 Cholo, M. C., Steel, H. C., Fourie, P. B., Germishuizen, W. A. \& Anderson, R. Clofazimine: current status and future prospects. J Antimicrob Chemother 67, 290-298, doi:10.1093/jac/dkr444 (2012).

25 Herold, T. et al. Elevated levels of IL-6 and CRP predict the need for mechanical ventilation in COVID-19. J Allergy Clin Immunol 146, 128-136 e124, doi:10.1016/j.jaci.2020.05.008 (2020).

26 Hwang, T. J. et al. Safety and availability of clofazimine in the treatment of multidrug and extensively drug-resistant tuberculosis: analysis of published guidance and meta-analysis of cohort studies. BMJ Open 4, e004143-e004143, doi:10.1136/bmjopen-2013-004143 (2014).

27 Sangana, R., Gu, H., Chun, D. Y. \& Einolf, H. J. Evaluation of Clinical Drug Interaction Potential of Clofazimine Using Static and Dynamic Modeling Approaches. Drug Metabolism and Disposition 46, 2632, doi:10.1124/dmd.117.077834 (2018).

28 Falzon, D. et al. World Health Organization treatment guidelines for drug-resistant tuberculosis, 2016 update. Eur Respir J 49, doi:10.1183/13993003.02308-2016 (2017).

29 Tang, S. et al. Clofazimine for the treatment of multidrug-resistant tuberculosis: prospective, multicenter, randomized controlled study in China. Clin Infect Dis 60, 1361-1367, doi:10.1093/cid/civ027 (2015). 
30 Dalcolmo, M. et al. Effectiveness and safety of clofazimine in multidrug-resistant tuberculosis: a nationwide report from Brazil. Eur Respir J 49, doi:10.1183/13993003.02445-2016 (2017).

31 Baik, J. \& Rosania, G. R. Molecular imaging of intracellular drug-membrane aggregate formation. Mol Pharm 8, 1742-1749, doi:10.1021/mp200101b (2011).

32 Morrison, N. E. \& Marley, G. M. The mode of action of clofazimine DNA binding studies. International journal of leprosy and other mycobacterial diseases : official organ of the International Leprosy Association 44, 133-134 (1976).

33 Lucas, C. et al. Longitudinal analyses reveal immunological misfiring in severe COVID-19. Nature 584, 463-469, doi:10.1038/s41586-020-2588-y (2020).

34 Ren, Y. R. et al. Clofazimine Inhibits Human Kv1.3 Potassium Channel by Perturbing Calcium Oscillation in T Lymphocytes. PLOS ONE 3, e4009, doi:10.1371/journal.pone.0004009 (2008).

35 <https://www.gilead.com/news-and-press/press-room/press-releases/2020/6/an-open-letter-fromdaniel-oday-chairman-ceo-gilead-sciences> (2020).

36 Weng, Z. et al. A simple, cost-effective but highly efficient system for deriving ventricular cardiomyocytes from human pluripotent stem cells. Stem Cells Dev 23, 1704-1716, doi:10.1089/scd.2013.0509 (2014).

37 Yuan, S. et al. SREBP-dependent lipidomic reprogramming as a broad-spectrum antiviral target. Nature Communications 10, 120, doi:10.1038/s41467-018-08015-x (2019).

38 Chu, H. et al. Comparative replication and immune activation profiles of SARS-CoV-2 and SARSCoV in human lungs: an ex vivo study with implications for the pathogenesis of COVID-19. Clin Infect Dis, doi:10.1093/cid/ciaa410 (2020).

39 Zhang, A. J. et al. SARS-CoV-2 infects and damages the mature and immature olfactory sensory neurons of hamsters. Clin Infect Dis, doi:10.1093/cid/ciaa995 (2020).

40 To, K. K. et al. Temporal profiles of viral load in posterior oropharyngeal saliva samples and serum antibody responses during infection by SARS-CoV-2: an observational cohort study. Lancet Infect Dis 20, 565-574, doi:10.1016/S1473-3099(20)30196-1 (2020).

$41 \mathrm{Kim}, \mathrm{D}$. et al. TopHat2: accurate alignment of transcriptomes in the presence of insertions, deletions and gene fusions. Genome Biol 14, R36, doi:10.1186/gb-2013-14-4-r36 (2013).

42 Liao, Y., Smyth, G. K. \& Shi, W. featureCounts: an efficient general purpose program for assigning sequence reads to genomic features. Bioinformatics 30, 923-930, doi:10.1093/bioinformatics/btt656 (2014). 
43 Yu, G., Wang, L. G., Han, Y. \& He, Q. Y. clusterProfiler: an R package for comparing biological themes among gene clusters. OMICS 16, 284-287, doi:10.1089/omi.2011.0118 (2012).

44 Shannon, P. et al. Cytoscape: a software environment for integrated models of biomolecular interaction networks. Genome Res 13, 2498-2504, doi:10.1101/gr.1239303 (2003).

45 Hoffmann, M. et al. SARS-CoV-2 Cell Entry Depends on ACE2 and TMPRSS2 and Is Blocked by a Clinically Proven Protease Inhibitor. Cel/ 181, 271-280.e278, doi:https://doi.org/10.1016/j.cell.2020.02.052 (2020).

46 Xie, X. et al. An Infectious cDNA Clone of SARS-CoV-2. Cell Host \& Microbe 27, 841-848.e843, doi:https://doi.org/10.1016/j.chom.2020.04.004 (2020).

47 Riva, L. et al. Discovery of SARS-CoV-2 antiviral drugs through large-scale compound repurposing. Nature, doi:10.1038/s41586-020-2577-1 (2020).

48 lanevski, A., Giri, A. K. \& Aittokallio, T. SynergyFinder 2.0: visual analytics of multi-drug combination synergies. Nucleic Acids Research 48, W488-W493, doi:10.1093/nar/gkaa216 (2020).

\section{Figures}




\section{Figure 1}

a

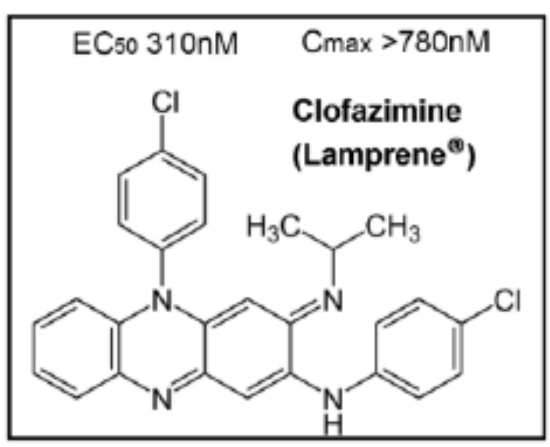

d

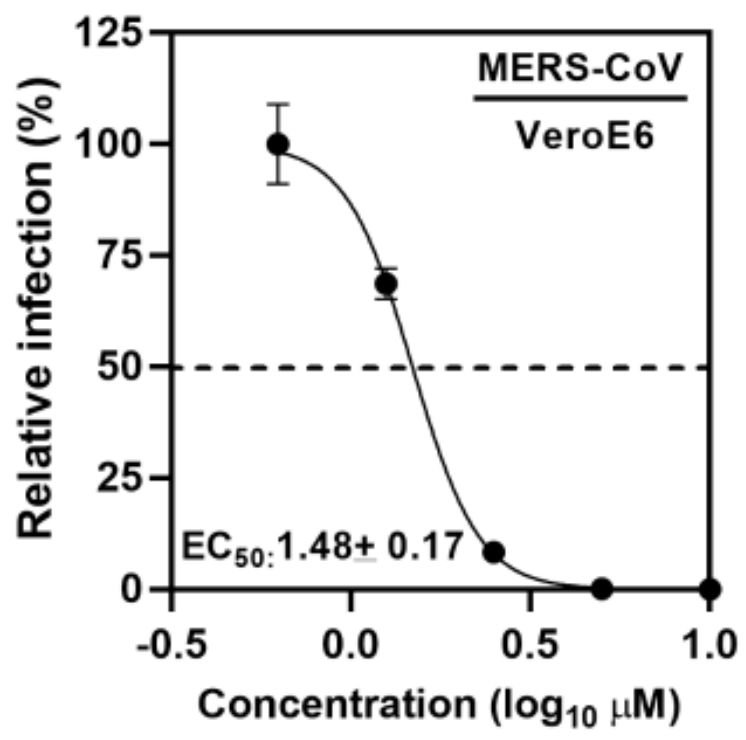

b

- Remdesivir ○ Clofazimine - DMSO

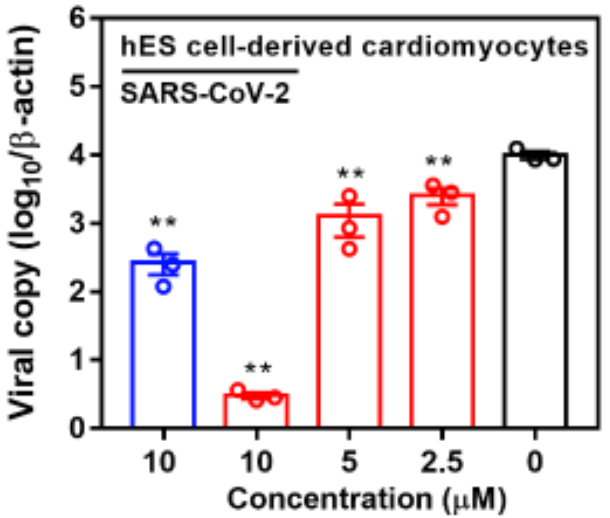

C

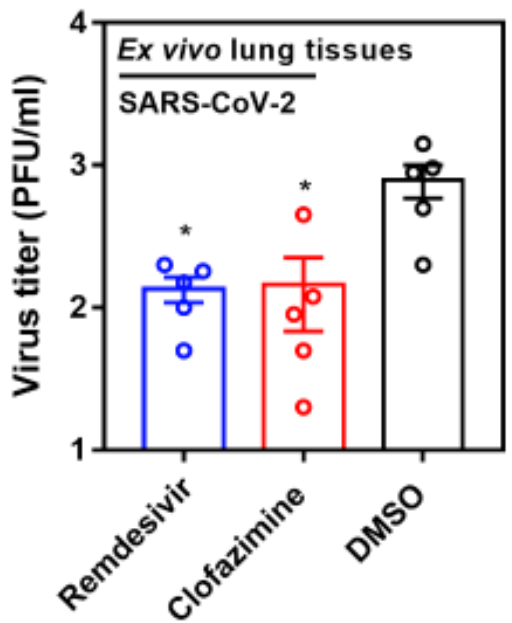

e
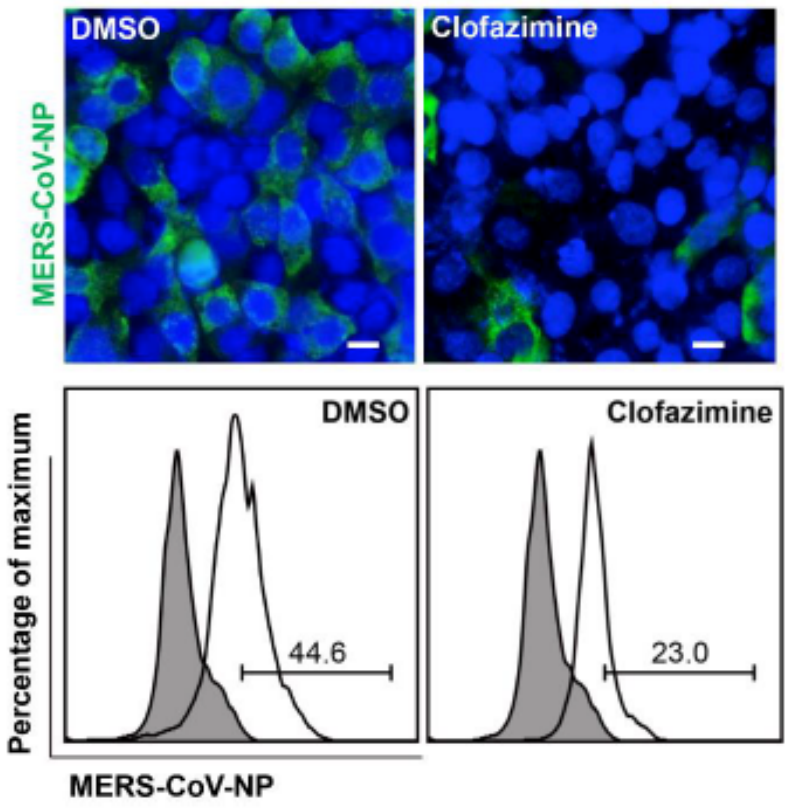

Figure 1

Clofazimine inhibits SARS-CoV-2 and MERS-CoV replication in human cellular models. (a) Chemical structure of clofazimine (brand name Lamprene) had an EC50 of $310 \mathrm{nM}$ against SARS-CoV-2 and peak serum concentration $\mathrm{Cmax}>780 \mathrm{nM}$ through oral administration in patients 13. (b) Clofazimine inhibited SARS-CoV-2 replication in human embryonic stem cells-derived cardiomyocytes that were infected by 0.1 $\mathrm{MOI}$ virus and treated with clofazimine $(10,5,2.5 \mu \mathrm{M})$ or $0.1 \%$ DMSO or remdesivir $(10 \mu \mathrm{M})$. Cell lysate 
were collected at the $24 \mathrm{hpi}$ with viral load determined by RT-qPCR assays. Data represent mean \pm SD for $n=3$ biological replicates. One-way AVONA when compared with DMSO group. ${ }^{*} p<0.01$. (c) Ex vivo human lung tissues were infected with SARS-CoV- 2 with an inoculum of $5 \times 105$ PFU. After two hours, the inoculum was removed before clofazimine $(10 \mu \mathrm{M})$ or remdesivir $(10 \mu \mathrm{M})$ or DMSO $(0.1 \%)$ was added and incubated for $24 \mathrm{~h}$. Supernatants were collected for quantification of viral titer by plaque assay. Error bars represent $S D$ for $n=5$ biological replicates. Student's t-test. ${ }^{*} p<0.05$. (d) Clofazimine inhibited MERS$\mathrm{CoV}$ replication in a dose-dependent manner. EC50 was achieved by plaque reduction assay on VeroE6 cells and plotted using logistic non-linear regression model (GraphPad). (e) MERS-CoV-infected Huh7 cells $(0.01 \mathrm{MOI})$ were treated with or without clofazimine $(5 \mu \mathrm{M})$ for $24 \mathrm{~h}$. Upper panel:

immunofluorescence staining of MERS-CoV-NP antigen (green), and Huh 7 cell nucleus (blue). Scale bar: $20 \mu \mathrm{m}$. Lower panel: MERS-CoV-NP positive cells quantitated by flow cytometry. The experiments were performed twice with representative images and quantifications shown. 


\section{Figure 2}

a
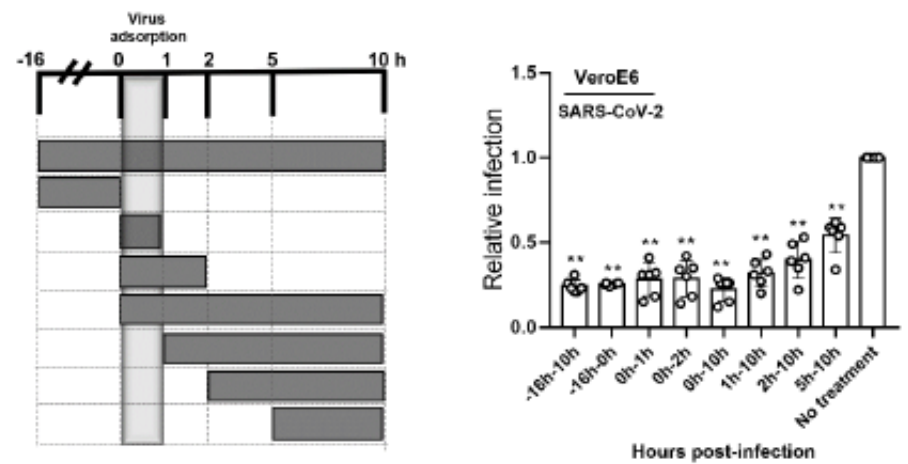

b

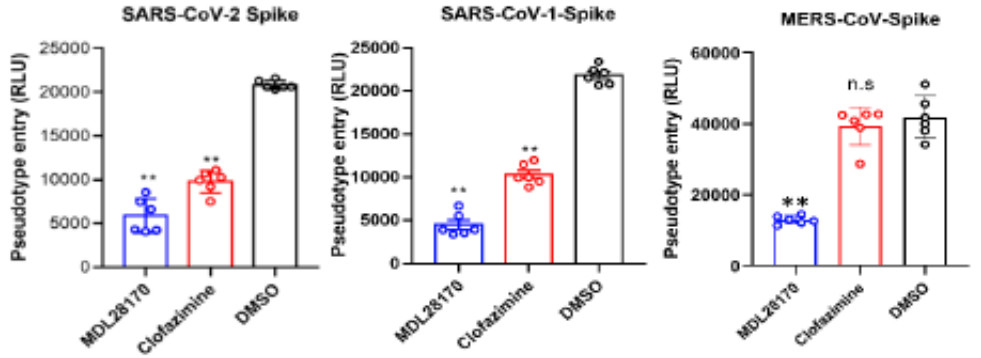

C

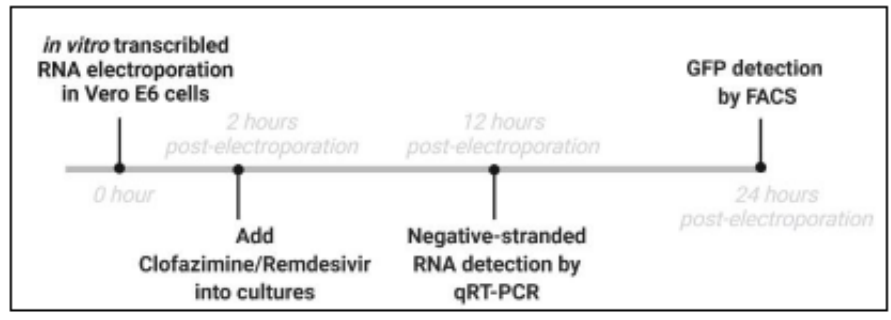

d

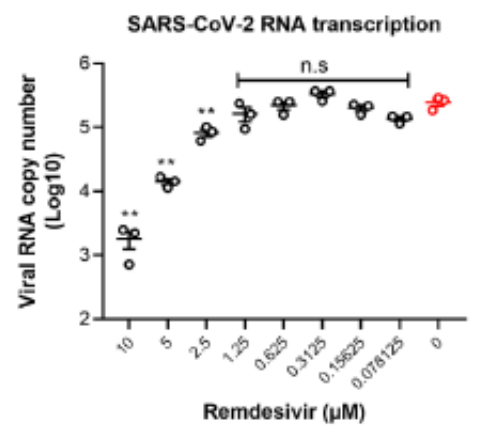

e

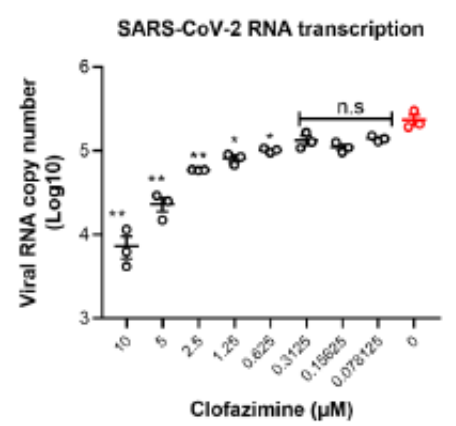

$\mathbf{f}$

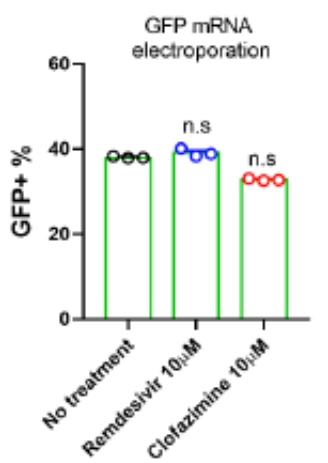

\section{Figure 2}

Clofazimine interferes with multiple steps of virus life cycle. (a) Time-of-addition assay. VeroE6 were inoculated with SARS-CoV-2 for $1 \mathrm{~h}$, and the inoculum was then removed. Cells were also incubated with clofazimine at a concentration of $5 \mu \mathrm{M}$ at time-points indicated. Infection at $10 \mathrm{hpi}$ was quantified by immunostaining for CoV NP. Data are normalized to the average of DMSO-treated wells for each corresponding time-point and are presented as mean \pm SD for $n=6$ independent experiments. Two-way 
ANOVA followed by Tukey post-hoc test was performed as statistical analysis. (b)VSV-based pseudotyped viral particles assay. VeroE6 cells were pre-treated with the indicated compounds $(2.5 \mu \mathrm{M})$ for $2 \mathrm{~h}$ and then infected with SARS-CoV-1 S or SARS-CoV-2 $\mathrm{S}$ or MERS-CoV S pseudotyped particles harboring firefly luciferase for additional $2 \mathrm{~h}$. Luciferase signal was quantified at $24 \mathrm{~h}$ post-inoculation. Error bars represent SEM for $n=6$ independent experiments. One-way ANOVA followed by Dunnett posttest was performed for statistical analysis. ${ }^{*} P \leq 0.05$, $* * P \leq 0.01$. MDL28170 was utilized as a positive control entry inhibitor. (c-f) In vitro transcribed viral RNA based on viral replication assay. VeroE6 cells were electroporated with in vitro transcribed viral RNA. At $2 \mathrm{~h}$ after seeding, the cells were treated with clofazimine (d) or remdesivir (e) at the indicated dose. Negative-stranded RNA was then quantified at 12 $h$ post-electroporation. Error bars represent SEM for $n=3$ independent experiments. GFP mRNA (f) was utilized as a negative control and its translation was not affected by remdesivir or clofazimine. Error bars represent SEM for $n=3$ independent experiments. ${ }^{*} \mathrm{P} \leq 0.05,{ }^{* *} \mathrm{P} \leq 0.01$. 
Figure 3

a

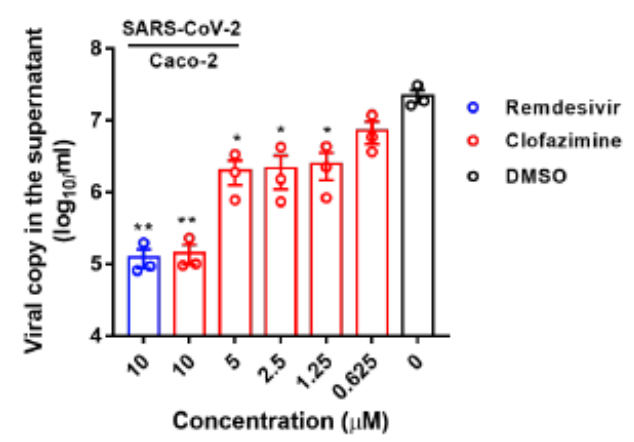

C
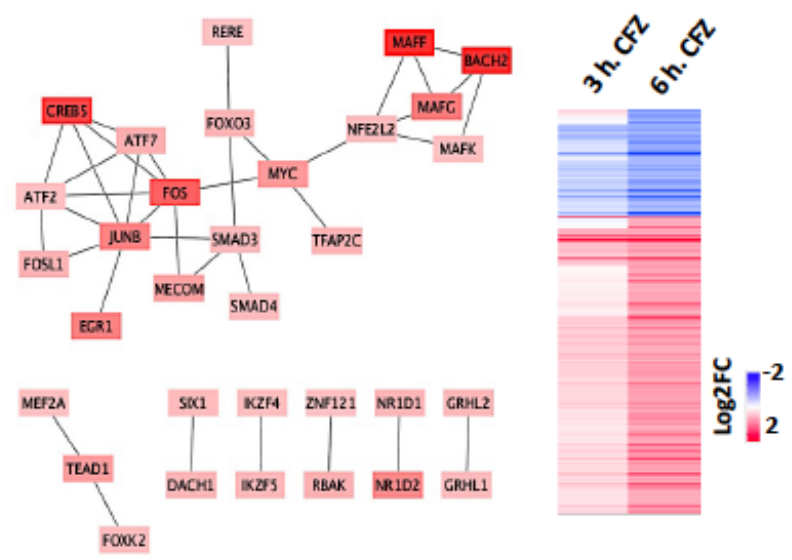

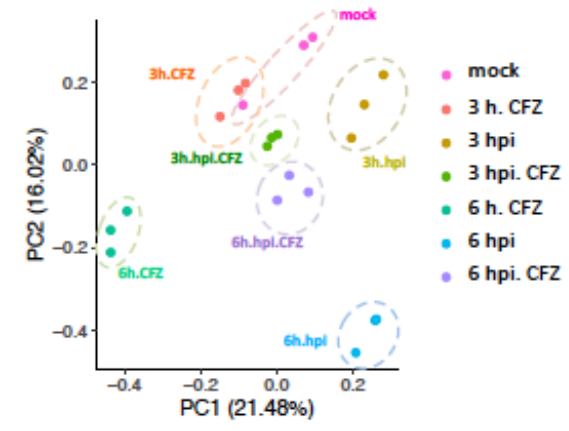

2

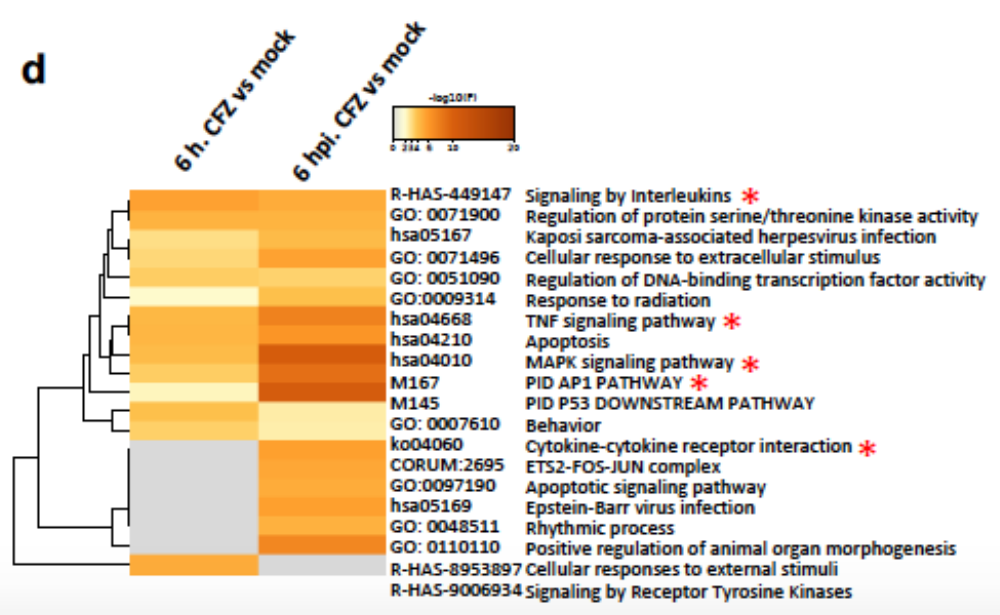

Figure 3

Transcriptional analysis of clofazimine treatment. (a) Caco-2 cells were infected with $0.1 \mathrm{MOI}$ of SARSCoV-2 before treated with the clofazimine, remdesivir and DMSO (0.1\%) at the indicated concentrations. Cell culture supernatant was collected at $48 \mathrm{hpi}$ and subjected to viral load determination by RT-qPCR analysis. One-way ANOVA as compared with the DMSO group. ${ }^{*} p<0.05,{ }^{*} p<0.01$. (b) PCA analysis of RNA-seq dataset after RPKM (Reads Per Kilobase of transcript per Million reads mapped) normalization 
on each gene expression level. Each dot represents one sample. CFZ = clofazimine treatment. Conditions include $3 \mathrm{hpi}$ and $6 \mathrm{hpi}$ of Caco-2 cells post infection, $\mathrm{MOI}=4$, with/without clofazimine treatment (CFZ) or CFZ treatment alone. (c) Heatmap of 197 transcription factors regulated by clofazimine treatment without infection (right), and known interactions among these transcription factors. (d) Top enriched pathways of significantly up-regulated genes ( $F D R<0.05$, fold change $>2$ ) compared between clofazimine treatment alone at $6 \mathrm{~h}$ versus mock-infected cells ( $6 \mathrm{~h} \mathrm{CFZ} \mathrm{vs} \mathrm{mock)} \mathrm{and} \mathrm{at} 6 \mathrm{hpi}$ under clofazimine treatment versus mock ( 6 hpi. CFZ vs mock). Pathway analysis was performed by Metascape ( ${ }^{\star}$ labels innate immunity related pathways). 


\section{Figure 4}

a

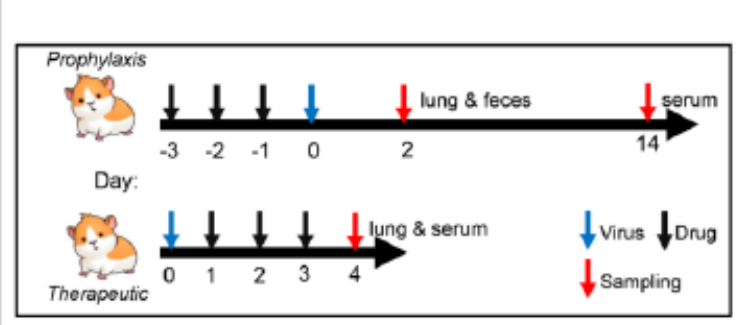

b

C
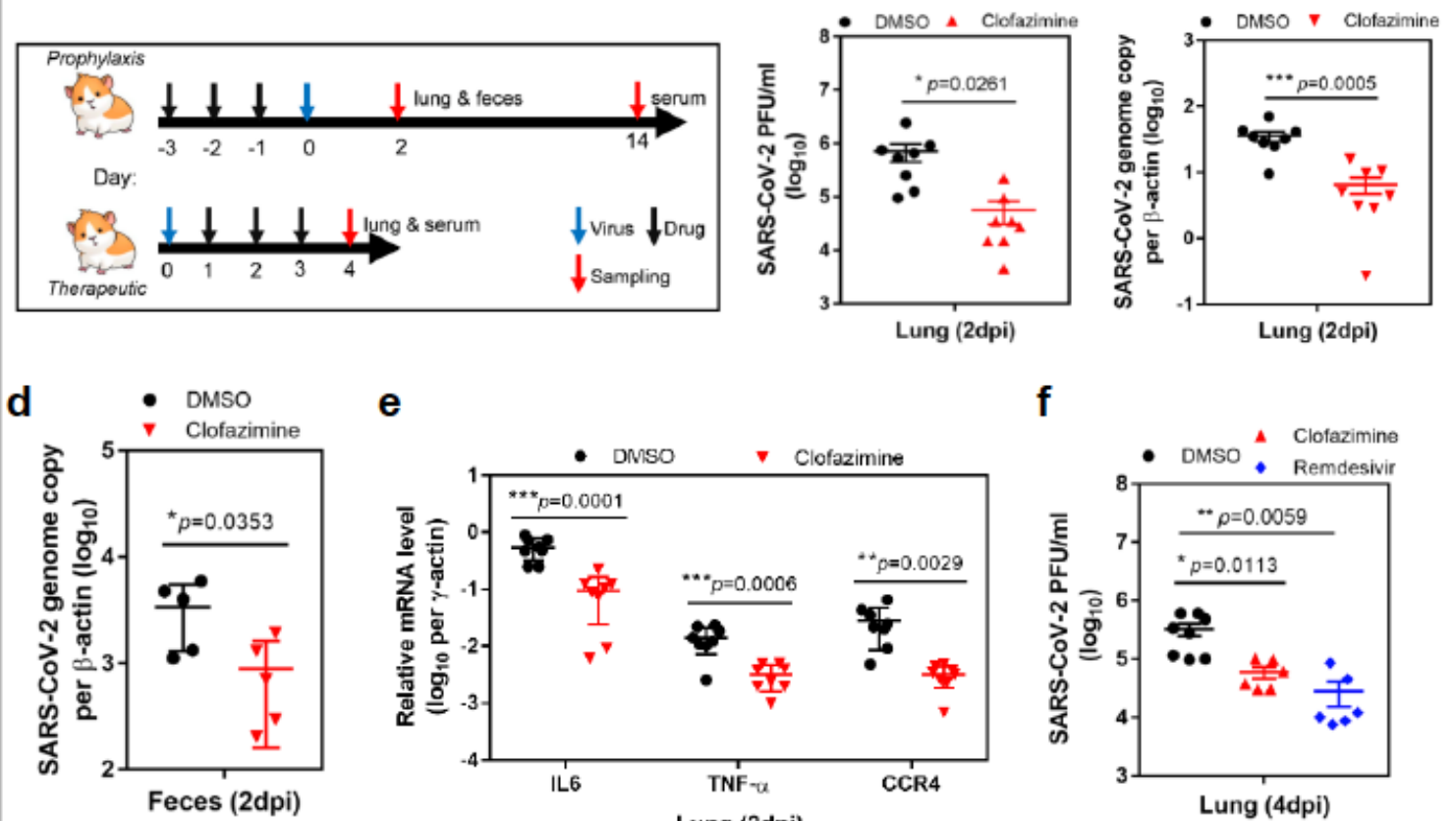

e

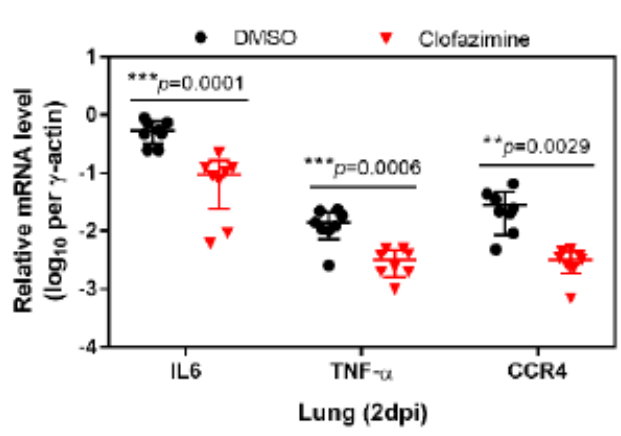

f
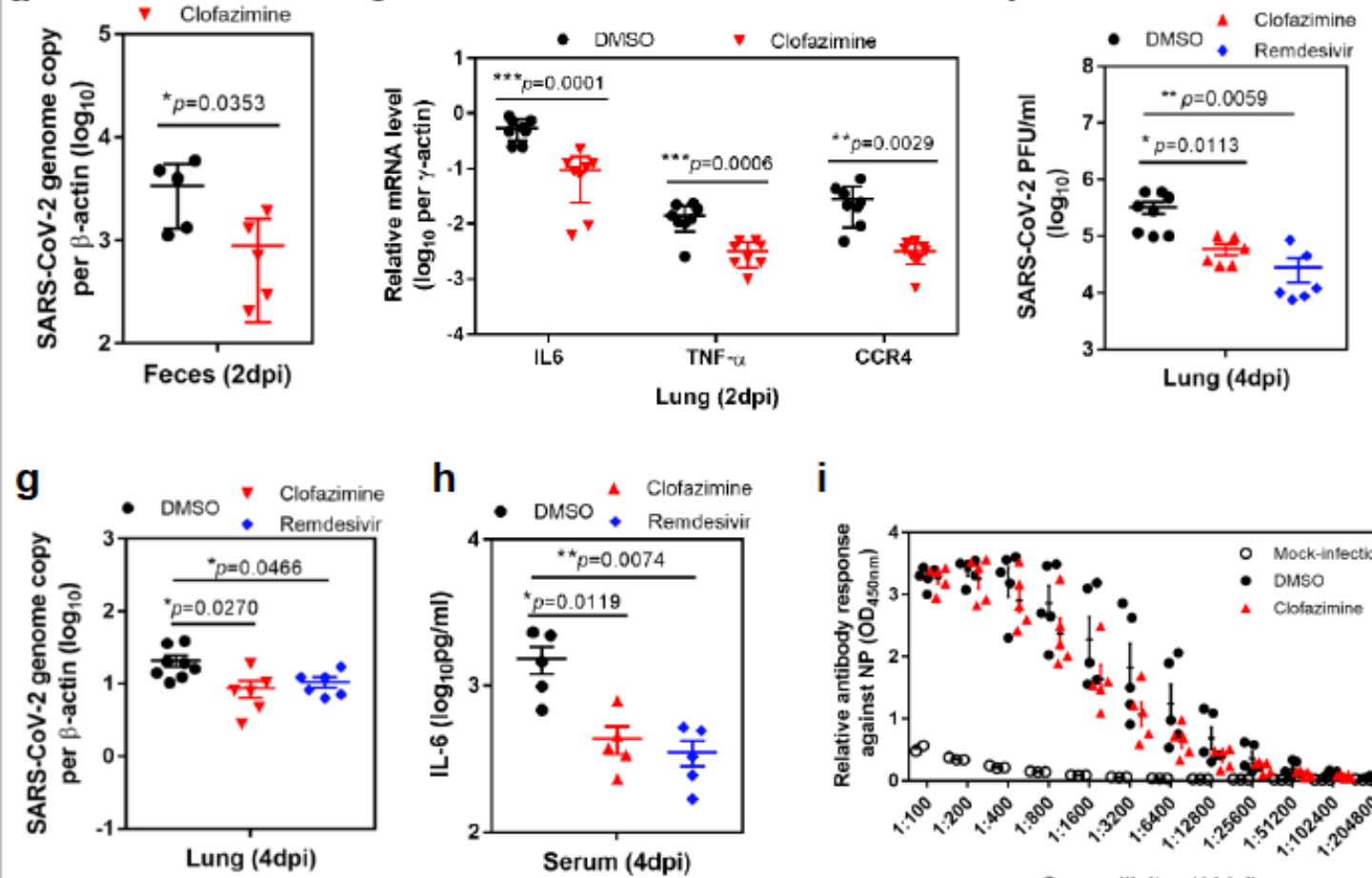

Serum (4dpi)

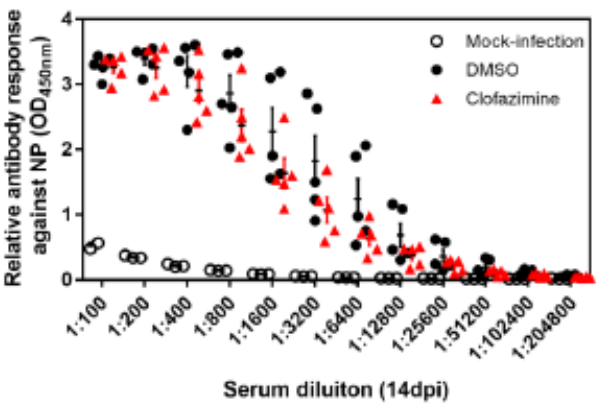

j

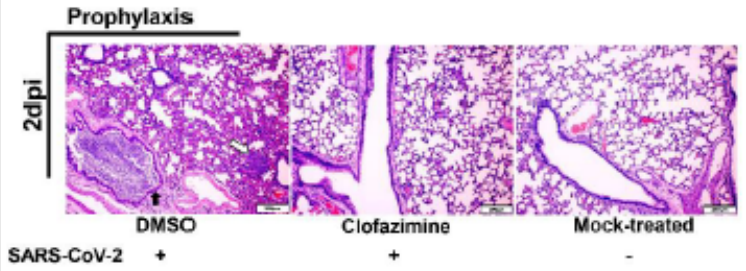

SARS-COV-2 +

$+$

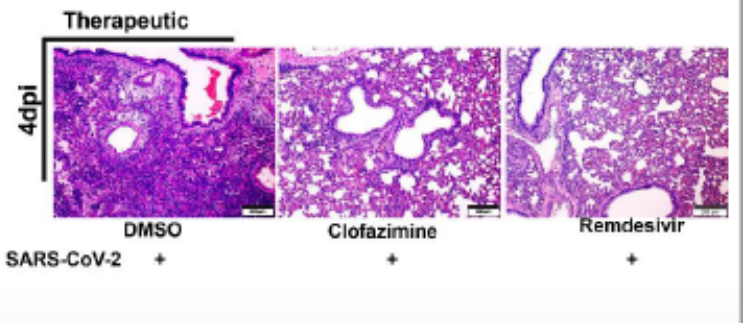

Figure 4

Prophylactic and therapeutic treatment with clofazimine reduces SARS-CoV-2 disease. (a) Experimental design: prophylactic treatment used oral administration of clofazimine given at $-3,-2$ and -1 dpi (25 $\mathrm{mg} / \mathrm{kg} / \mathrm{day}$ ), followed by virus challenge at 0dpi through intranasal route (105 PFU/hamster); therapeutic post-exposure administration of clofazimine was performed at $1 \mathrm{dpi}$ applying the same drug dosage and virus inoculum. Tissue and serum samples were collected at indicated days post-infection. Remdesivir 
(15 mg/kg) was included as a positive control in the therapeutic regimen through intraperitoneal injection. ( $b$ and c) Prophylactic experiments: viral yield in the hamster lung tissue $(n=8)$ were harvested at $2 \mathrm{dpi}$ and titrated by plaque assay and RT-qPCR assay (normalized by $\beta$-actin), respectively. Student's ttest. (d) Hamster feces, freshly collected at 2 dpi, were subjected to SARS-CoV-2 viral copy detection by RT-qPCR assays ( $n=5)$. Student's t-test. (e) Representative chemokine and cytokine levels of the lung tissue homogenates of the indicated groups $(n=8)$ at $2 \mathrm{dpi}$. The results are shown as mean value $\pm S D$. Student's t-test within each gene's group. ( $f$ and $g$ ) Therapeutic experiments: viral yield in the hamster lung tissue $(n=5)$ were harvested at $4 \mathrm{dpi}$ and titrated by plaque assay and RT-qPCR assay (normalized by $\beta$-actin), respectively. One-way ANOVA when compared with the control group (1\% DMSO in corn oil by oral administration). (h) The disease severity marker L- 6 level in hamster serum were quantified by ELISA at 4 dpi. N=5. One-way ANOVA. (i) Hamster exhibited normal humoral immune response after SARS-CoV2 infection and clofazimine treatment. Enzyme immunoassay (EIA) for immunoglobulin G against SARSCoV-2 nucleoprotein was performed in hamster sera of the indicated groups collected at $14 \mathrm{dpi}$. The sera were serially diluted before adding to the NP-coated ELISA plate. $\mathrm{N}=5$ for each group. (j) Left panel (prophylaxis): representative DMSO-treated hamster lungs showed severe bronchiolar cell death with massive cell debris filling the lumen (solid arrow). Alveolar wall thickened with alveolar exudation were observed (open arrow). Lungs of clofazimine-treated and mock-infected groups showed no apparent pathological changes. Right panel (therapeutic): representative DMSO-treated lung section showed large area of lung consolidation with alveolar infiltration. Bronchial wall showed luminal haemorrhagic exudation. Clofazimine or remdesivir-treated lung exhibited mild degree of alveolar wall thickening and capillary congestion with intact epithelium and without luminal cell debris. ${ }^{*} p<0.05,{ }^{* *} p<0.01,{ }^{* *} p<0.001$.

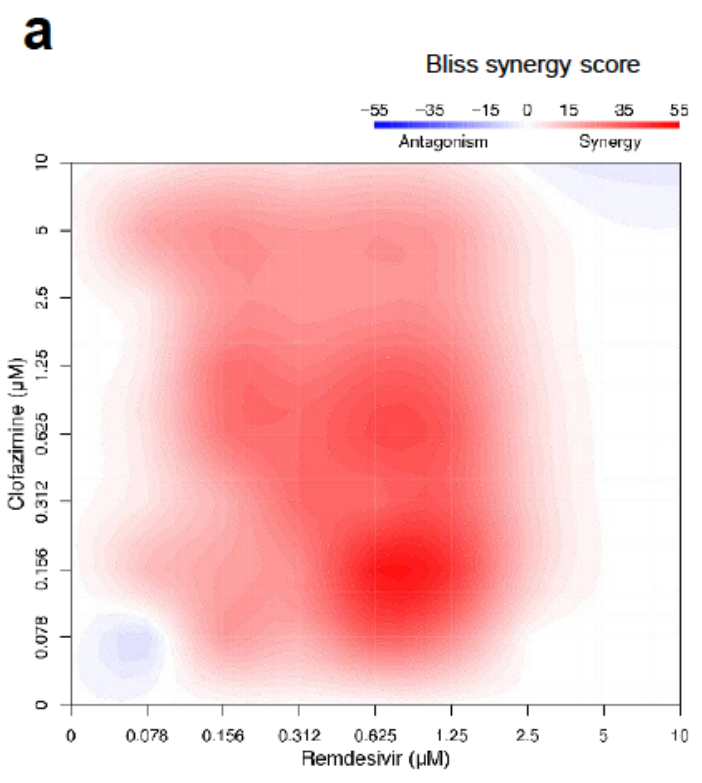

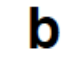

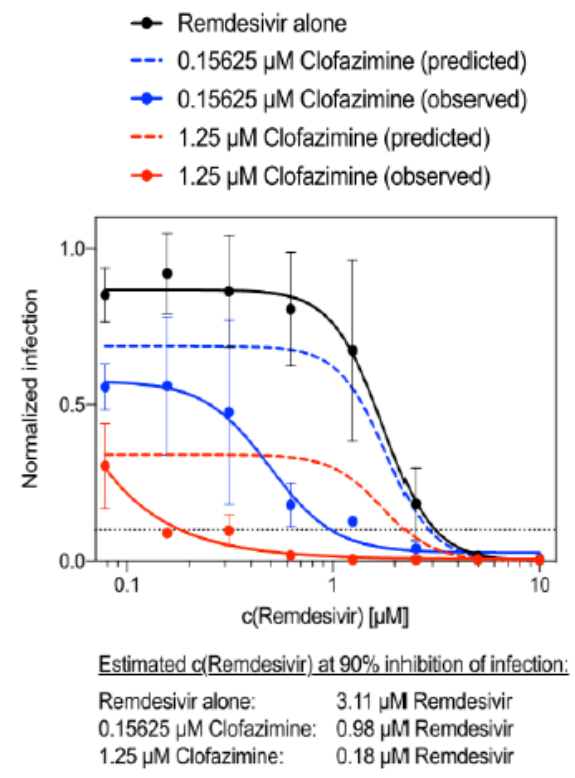

C

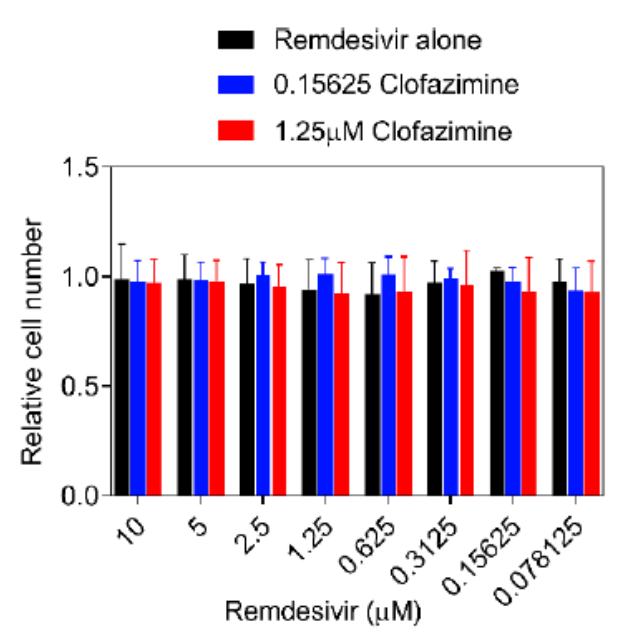




\section{Figure 5}

Clofazimine exhibits antiviral synergy with remdesivir. VeroE6 cells were pre-treated for $16 \mathrm{~h}$ with increasing concentrations of the indicated compound and then infected with SARS-CoV-2 at a MOI of 0.01 . Thirty hours after infection, cells were fixed and analysed by immunofluorescence imaging. For each condition, the percentage of infection was calculated as the ratio of the number of infected cells stained for coronavirus NP to the number of cells stained with DAPI. (a) Topographic two-dimensional map of synergy scores determined in synergy finder 48 . Color gradient indicates synergy score (redhighest score). X-axis: remdesivir up to $10 \mu \mathrm{M}$, y-axis: clofazimine up to $10 \mu \mathrm{M}$. (b) Dose response analysis of remdesivir alone (black) and in combination with $0.15625 \mu \mathrm{M}$ (blue) or $0.625 \mu \mathrm{M}$ (red) clofazimine. The observed compound activities are represented by solid lines, while the predicted additive combinatorial activities are indicated by dashed lines. $90 \%$ inhibition of infection is denoted by a dotted black line. Data are normalized to mean values for DMSO-treated wells and represent mean \pm SEM. of $n=$ 2 independent experiments.

\section{Supplementary Files}

This is a list of supplementary files associated with this preprint. Click to download.

- Figs1.pdf

- FigS2.pdf 